\title{
Cantilever-Enhanced Photoacoustic Spectroscopy of Radioactive Methane
}

\section{Larnimaa, S.}

\section{IEEE}

2020-05

Larnimaa , S , Karhu , J , Tomberg , T , Genoud, G , Hieta , T, Metsälä, M , Halonen , L \& Vainio, M 2020 , Cantilever-Enhanced Photoacoustic Spectroscopy of Radioactive Methane . in 2020 Conference on Lasers and Electro-Optics (CLEO) ., 9193205 , Conference Proceedings - Lasers and Electro-Optics Society Annual Meeting-LEOS , IEEE , 2020 Conference on Lasers and Electro-Optics, San Jose , United States , 10/05/2020 . < https://ieeexplore.ieee.org/document/9193205 >

http://hdl.handle.net/10138/337975

acceptedVersion

Downloaded from Helda, University of Helsinki institutional repository.

This is an electronic reprint of the original article.

This reprint may differ from the original in pagination and typographic detail.

Please cite the original version. 


\title{
Cantilever-Enhanced Photoacoustic Spectroscopy of Radioactive Methane
}

\author{
Santeri Larnimaa ${ }^{1}$, Juho Karhu ${ }^{1}$, Teemu Tomberg ${ }^{1}$, Guillaume Genoud ${ }^{2}$, Tuomas Hieta ${ }^{3}$, Markus Metsälä ${ }^{1}$, \\ Lauri Halonen ${ }^{1}$, Markku Vainio ${ }^{1,4}$ \\ 1. Department of Chemistry, University of Helsinki, Helsinki, Finland \\ 2. Centre for Metrology MIKES, VTT Technical Research Centre of Finland Ltd., Finland \\ 3. Gasera Ltd., Turku, Finland \\ 4. Photonics Laboratory, Physics Unit, Tampere University, Tampere, Finland \\ Authore-mail address: markku.vainio@helsinki.fi
}

\begin{abstract}
We report the first high-resolution spectroscopy study of radiocarbon methane, ${ }^{14} \mathrm{CH}_{4}$. Several absorption lines of the fundamental vibrational band $v_{3}$ were measured using a continuous-wave mid-infrared optical parametric oscillator with cantilever-enhanced photoacoustic spectroscopy. ( 2020 The Authors
\end{abstract}

\section{Introduction}

The standard method for sensitive measurements of long-lived radioactive isotopes, such as ${ }^{14} \mathrm{C}$, is accelerator mass spectrometry (AMS). The AMS facilities are indispensable for applications like radiocarbon dating, but too expensive and massive for in-situ measurements. Laser spectroscopy is a promising solution for isotope-selective detection of gas-phase compounds in applications that require low-cost instrumentation and on-site measurement capability. The recent work on laser spectroscopy of ${ }^{14} \mathrm{CO}_{2}$ has led to impressive detection limits even below the natural ${ }^{14} \mathrm{C}$ abundance [1-3]. This encourages us to develop similar techniques also for other radiocarbon compounds, such as ${ }^{14} \mathrm{CH}_{4}$. The potential applications of optical detection of ${ }^{14} \mathrm{CH}_{4}$ include in-situ monitoring of radioactive gas emissions at decommissioning sites of light water nuclear reactors, as well as measurements of the biofraction of methane fuel mixtures.

A high resolution spectrum is an essential prerequisite for the development of optical analysis instrumentation for radiocarbon methane. We reported the first measurements of the optical absorption spectrum of ${ }^{14} \mathrm{CH}_{4}$ in 2019 [4]. Those measurements were made possible by a novel photoacoustic optical frequency comb method, which enabled us to assign some of the strongest lines of the antisymmetric $\mathrm{CH}$-stretching vibrational band $v_{3}$. Here, we extend our prior work by reporting the first high-resolution study of the same band. We have used a customized narrowlinewidth continuous-wave optical parametric oscillator (CW OPO) together with cantilever-enhanced photoacoustic spectroscopy (CEPAS) to improve the spectral resolution and detection sensitivity by approximately a factor of 10 and 30, respectively. These improvements have made it possible to observe several ${ }^{14} \mathrm{CH}_{4}$ lines that were previously too weak to be measured, or were covered by strong ${ }^{12} \mathrm{CH}_{4}$ absorption. The line positions can be determined with high precision, which helps us to identify and characterize lines that are suitable for trace-gas detection applications.

\section{Experiment}

Our light source is a home-built CW OPO [5], which is tuneable from 2700 to $3400 \mathrm{~nm}\left(2940-3710 \mathrm{~cm}^{-1}\right)$. The $\mathrm{CW}$ OPO output power is more than $1 \mathrm{~W}$ over the entire wavelength tuning range and the linewidth is approximately $1 \mathrm{MHz}$. The mid-infrared wavelength is locked to a wavelength meter (Bristol 771B) for precise computer-controlled high-resolution scans.

The CW OPO output beam is directed to a CEPAS cell (Gasera PA201), which contains the gas sample prepared by Quotient Bioresearch. The CEPAS cell has a volume of only $8 \mathrm{ml}$, which is ideal for samples with limited availability, such as the radiocarbon methane sample. The gas mixture has a total methane concentration of about $100 \mathrm{ppm}$ in nitrogen, with about $1 \mathrm{ppm}$ of the ${ }^{14} \mathrm{CH}_{4}$ isotopologue. The sample pressure during the measurements is typically lowered to $200 \mathrm{mbar}$, in order to reduce the pressure broadening and thus to better resolve the shapes of the methane lines, which are split into closely packed line components due to Coriolis interaction. Further improvement of the spectral selectivity and signal-to-noise ratio is obtained by using wavelength modulation with $2 \mathrm{f}$ detection. The modulation frequency $(30 \mathrm{~Hz})$ and amplitude were optimized for CEPAS detection [6] and the required modulation was performed via the CW OPO pump laser. 


\section{Results and outlook}

An example of a 2 f CEPAS spectrum of the $R(3)$ line of the $v_{3}$ band of radiocarbon methane is shown in Fig. 1. The figure demonstrates the high spectral resolution, which makes it possible to resolve the Coriolis components. These components are easier to identify from the right-hand side of the figure, which shows the absorption spectrum constructed from the measured $2 \mathrm{f}$ spectrum. The noise-equivalent detection limit of the measurement is 3 parts per billion, which is a factor of 30 better than in the previous measurements of the same line [4]. We have measured in total 43 line components, 17 of which are new, previously unobserved components. In particular, the $\mathrm{P}$ branch of the $v_{3}$ band is observed with high signal-to-noise ratio for the first time. The high quality of data makes it possible to the better asses the band parameters, as well as to identify optimal spectral regions for applications like radioactive gas emissions monitoring [7].
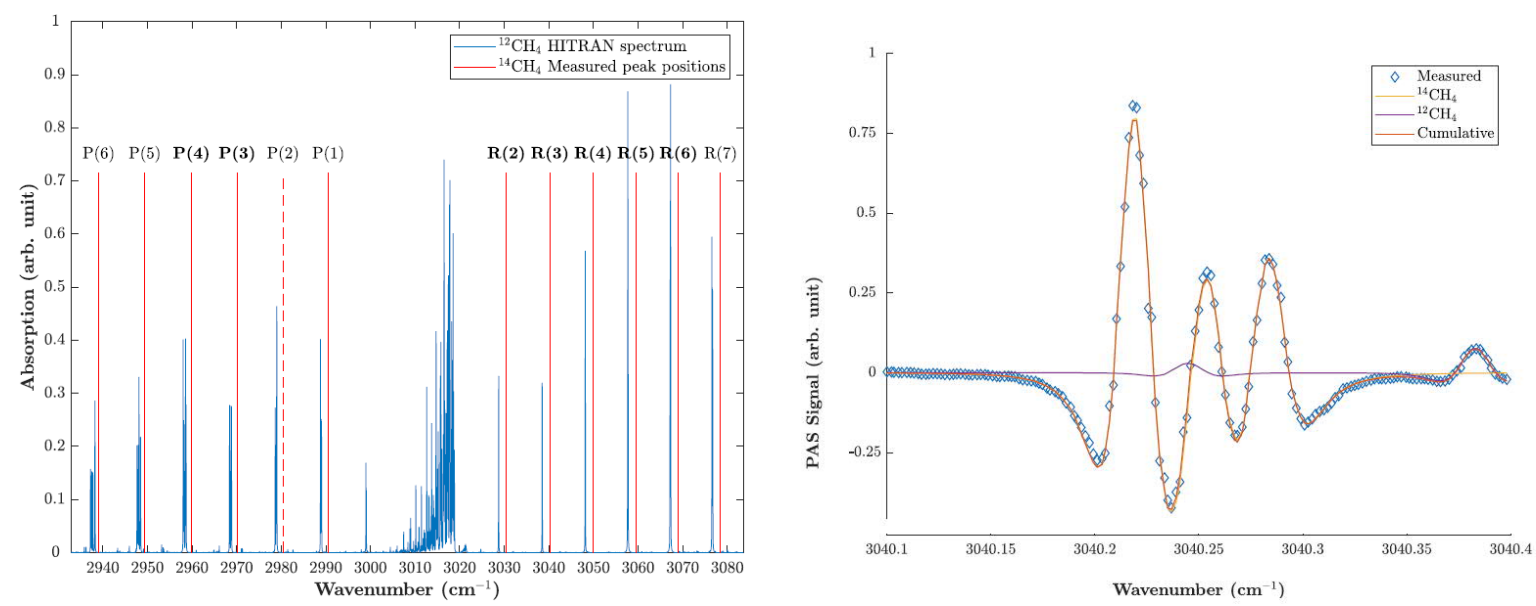

Fig. 1. Left: Summary of the measured radiocarbon methane lines, together with the already known ${ }^{12} \mathrm{CH}_{4}$ spectrum. Right: An example of the $2 \mathrm{f}$ CEPAS spectrum of line R(3), which consists of three line components. The fit also includes a couple of weak ${ }^{12} \mathrm{CH}_{4}$ lines.

Acknowledgements: We acknowledge the financial support of the Academy of Finland (Grant 314363).

\section{References}

[1] I. Galli, S. Bartalini, R. Ballerini, M. Barucci, P. Cancio, M. De Pas, G. Giusfredi, D. Mazzotti, N. Akikusa, and P. De Natale, "Spectroscopic detection of radiocarbon dioxide at parts-per-quadrillion sensitivity," Optica 3, 385 (2016).

[2] G. Genoud, M. Vainio, H. Phillips, J. Dean, and M. Merimaa, "Radiocarbon dioxide detection based on cavity ring-down spectroscopy and a quantum cascade laser," Opt. Lett. 40, 1342 (2015)

[3] A.J. Fleisher, D.A. Long, Q. Liu, Q, L. Gameson, and J.T. Hodges, “Optical Measurement of Radiocarbon below Unity Fraction Modern by Linear Absorption Spectroscopy” J. Phys. Chem. Lett. 8, 4550 (2017).

[4] J. Karhu, T. Tomberg, F.S. Vieira, G, Genoud, V. Hänninen, M. Vainio, M. Metsälä, T. Hieta, S. Bell, and L. Halonen, "Broadband photoacoustic spectroscopy of ${ }^{14} \mathrm{CH}_{4}$ with a high-power mid-infrared optical frequency comb," Opt. Lett. 44, 1142 (2019).

[5] M. Vainio, J. Peltola, S. Persijn, F. Harren, and L. Halonen, "Thermal effects in singly resonant continuous-wave optical parametric oscillators," Appl. Phys. B 94, 411 (2009).

[6] T. Tomberg, M. Vainio, T. Hieta and L. Halonen, "Sub-parts-per-trillion level sensitivity in trace gas detection by cantilever-enhanced photoacoustic spectroscopy," Sci. Rep. 8, 1848 (2018)

[7] G. Genoud, J. Lehmuskoski, S. Bell, V. Palonen, M. Oinonen, M-L. Koskinen-Soivi, and M. Reinikainen, "Laser Spectroscopy for Monitoring of Radiocarbon in Atmospheric Samples," Anal. Chem. 91, 12315 (2019).

(C) 2021 IEEE. Personal use of this material is permitted. Permission from IEEE must be obtained for all other uses, in any current or future media, including reprinting/republishing this material for advertising or promotional purposes, creating new collective works, for resale or redistribution to servers or lists, or reuse of any copyrighted component of this work in other works. 